\title{
A stochastic coverage model for erosion events caused by the intersection of burnt forest and convective thunderstorms
}

\author{
O.D. Jones, ${ }^{a, c}$ P. Nyman ${ }^{b, c}$ and G.J. Sheridan ${ }^{b, c}$ \\ ${ }^{a}$ The University of Melbourne, Department of Mathematics and Statistics, Melbourne, Australia \\ ${ }^{b}$ The University of Melbourne, School of Forest and Ecosystem Science, Melbourne, Australia \\ ${ }^{c}$ The Bushfire Cooperative Research Centre, East Melbourne, Victoria, Australia \\ Email: odjones@unimelb.edu.au
}

\begin{abstract}
Soil erosion events following fire can wash sediment and ash into streams and reservoirs, contaminating water supplies for cities and towns. These risks are real, yet difficult to quantify, constraining the optimal selection of preventative and remedial options such as prescribed burning and investment in water treatment infrastructure. Non-stationary climate and fire regimes resulting from climate change add to this difficulty. What is the chance of a water supply becoming unusable due to fire? Will this increase with climate change? Will prescribed burning increase or decrease this risk? Answering these questions is challenging because both fire and rainfall regimes are already complex processes to model individually. Considering the interaction between these two processes substantially increases the complexity of the modeling problem.
\end{abstract}

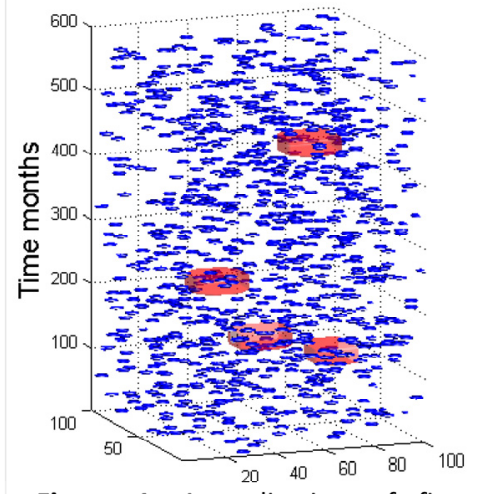

Figure 1. A realization of fire events (red) and storm events (blue) in space and time, illustrating the overlap between these processes

The model outlined in this paper is based on the premise that highmagnitude erosion events following fire result from the spatial and temporal intersection of burnt areas and high-intensity rainfall events. In this new model we consider fires and storms as independent stochastic processes with properties of spatial extent, temporal duration, and frequency of occurrence. This is illustrated in Figure 1, where we have superimposed realizations of fire and storm processes. Here the $(x, y)$-axes give the spatial extent and the vertical axis gives duration. The volume of intersection of the two processes (shown by the overlap of the large and small discs) gives a measure of hazard of high-magnitude erosion events, and we can quantify how it changes in response to changing fire and climate regimes.

Let the set $\Omega$ represent the catchment for a single year, then we are interested in the "risk set"

$R=\Omega \cap$ burnt area $\times$ duration $\cap$ stormy area $\times$ duration.

Here $R$ has dimensions $\mathrm{km}^{2} \times$ years. The duration of a fire is the time it takes for the vegetation to recover (a couple of years), rather than the time the fire is active (a couple of days). The volume of $R$, that is $\|R\|$, represents the annual area where burn and rainfall satisfy the conditions required for high-magnitude erosion events to occur in a catchment where post-fire response thresholds are known. Let $\lambda=$ fire event rate (per unit area and unit time); $\mu=$ storm event rate (per unit area and unit time); $\alpha=\mathrm{E} \|$ fire event $\|$ (in $\mathrm{km}^{2} \times$ years); and $\beta=\mathrm{E} \|$ rainfall event $\|$ (in $\mathrm{km}^{2} \times$ years). Given these definitions, we use the mathematics of coverage processes to show that

$$
\mathrm{E}\|R\|=\|\Omega\|\left(1-e^{-\lambda \alpha}\right)\left(1-e^{-\mu \beta}\right) .
$$

In addition to deriving this result we obtain estimates for $\lambda, \mu, \alpha$ and $\beta$, and consider the effect on $\mathrm{E}\|R\|$ of changing these parameters.

Keywords: $\quad$ Post-fire erosion, water quality, risk, wildfire, prescribed fire, climate change 


\section{INTRODUCTION}

Erosion events following fire can wash sediment and ash into streams and reservoirs, contaminating water supplies for cities and towns (Nyman, et al., 2011, Smith, et al., 2011). These risks are real (e.g. White, et al., 2006) yet difficult to quantify, constraining the optimal selection of preventative and remedial options such as prescribed burning and investment in water treatment infrastructure. Non-stationary rainfall and fire regimes resulting from climate change add to this difficulty. What is the chance of a water supply becoming unusable due to fire? Will this increase with climate change? Will prescribed burning increase or decrease this risk?

A key element of this problem involves capturing the nature of the interaction between the causes: fires and rainfall events. The frequency and size of fires is highly variable, depending on climatic conditions in a particular year. Following fire we have a "window of risk" (Prosser and Williams, 1998) for several years within which severe erosion events may occur, depending on whether a storm event of sufficient magnitude occurs within the burnt areas. When a storm does occur in a burnt area, the magnitude of the erosion event is dependent on the landscape vulnerability, which comprises many factors including soil properties, topography, fire severity (hydrological perturbation) and level of recovery. Over time, the frequency and magnitude of impacts on water resources is a function of cause (fire and storms) and landscape vulnerability.

Fire and erosion are frequently modeled separately. In a few cases erosion modeling efforts have been specifically targeted at burnt landscapes (Cannon, et al., 2010, Robichaud, et al., 2007), though with the focus on predicting erosion rates after the landscape has been burnt, rather than assessing the combined impact of fire and rainfall on erosion. To our knowledge, Istanbulluoglu, et al. (2004) is the only existing attempt at modeling long term fire related erosion rates which incorporates stochastic fire and rainfall events. In their model, temporal dynamics of vegetation coverage and soil depth are modeled in some detail, but spatial variation is ignored.

The physical modeling of fires, rainfall and subsequent erosion events is potentially a very complex undertaking, requiring detailed deterministic fire and erosion models with many parameters and time-series of forcing inputs. Application of this modeling approach across landscapes is constrained by the availability of the high-resolution data required to fit the models. The deterministic representation of fire and erosion processes can result in very high epistemic uncertainties, due to the large number parameters and modeling steps. These uncertainties in turn can obscure the effects of key elements within the system, such as the frequency of fire and rainfall events.

In this paper we ask the question, "What is the first-order effect of the interaction between fires and storms?" When assessing changes in risk as a result of different fire regimes and/or climate change, we argue that the most important property of the system is the "volume" of the intersection in space and time of burnt areas and storms. The modeling focus should therefore be directed at the overlap between fire and storm events, rather than at the geophysical processes that drive them individually. We propose a novel method to quantify the size of this intersection as a function of the regional fire regime and the local rainfall properties. We then apply the model to SE Australia to illustrate how parameters can be obtained from readily available data on fire and rainfall regimes.

\section{THE MODEL}

\subsection{Model development}

The term coverage process refers to any stochastic process consisting of a number of sets, usually in some Euclidean space $\mathrm{R}^{d}$, where we are interested in the volume of some fixed set $\Omega$ which is covered by these random sets. A particular type of coverage process is the germ-grain model, in which the random sets are generated by taking a Poisson process (the germs) and then at each point centering iid random sets (the grains).

Our model uses two independent germ-grain processes, one for storms and the other for fires. Our space will be $\mathrm{R}^{3}$, where the first two dimensions are space $\left(\mathrm{km}^{2}\right)$ and the third is time (years). The set $\Omega$ represents the catchment for a single year, and we are interested in the "risk set"

$$
R=\Omega \cap \text { burnt area } \times \text { duration } \cap \text { stormy area } \times \text { duration }
$$

Here $R$ has dimensions $\mathrm{km}^{2} \times$ years. Our fire process will model fire events as patches of disturbance, and our storm process will model high-intensity storm cells, the combination of which is known to have the 
potential to cause high-magnitude erosion events. Here the duration of a fire is the time it takes for the vegetation to recover (a couple of years), rather than the time the fire is active (a couple of days). (So the fire duration is really the fire recovery time.) Thus we can interpret the volume of $R$, that is $\|R\|$, as the erosion hazard due to the overlap between storms and burnt areas.

We need some notation to describe our storm and fire processes

$$
\begin{aligned}
& \lambda=\text { fire event rate (per unit area and unit time) } \\
& \mu=\text { storm event rate (per unit area and unit time) } \\
& \alpha=\mathrm{E} \| \text { fire event } \|\left(\text { in } \mathrm{km}^{2} \times \text { years }\right) \\
& \beta=\mathrm{E} \| \text { rainfall event } \|\left(\text { in } \mathrm{km}^{2} \times \text { years }\right)
\end{aligned}
$$

Given these definitions, our main result for this section is that

$$
\mathrm{E}\|R\|=\|\Omega\|\left(1-e^{-\lambda \alpha}\right)\left(1-e^{-\mu \beta}\right) .
$$

Note that this formula only requires the expected size and rate of fires and storms, and does not depend on their shape. We will use this result to explore the impact of changing fire and storm behaviour on highmagnitude erosion events.

It is also possible to say something about how variable $R$ is. Let $A$ be a typical fire event centered at the origin, and $B$ a typical storm event centered at the origin (recall that we model fire and storm events as iid sequences). Define $\alpha(x)=\mathrm{E}\|(x+A) \cap A\|$ and $\beta(x)=\mathrm{E}\|(x+B) \cap B\|$, where $x+A$ is just the set obtained by adding $x$ to each element of $A$. Note that while $\alpha=\alpha(0)=\mathrm{E}\|A\|$ does not depend on the shape of $A, \alpha(x)$ does when $x \neq 0$. We have

$$
\begin{aligned}
\operatorname{Var}\|R\|=\int_{\Omega} \int_{\Omega} & \left(\left(1-e^{-\lambda \alpha(0)}\right)^{2} e^{-2 \mu \beta(0)}\left(e^{\mu \beta(x-y)}-1\right)\right. \\
& +\left(1-e^{-\mu \beta(0)}\right)^{2} e^{-2 \lambda \alpha(0)}\left(e^{\lambda \alpha(x-y)}-1\right) \\
& \left.+e^{-2(\lambda \alpha(0)+\mu \beta(0))}\left(e^{\lambda \alpha(x-y)}-1\right)\left(e^{\mu \beta(x-y)}-1\right)\right) d x d y .
\end{aligned}
$$

Proofs for these results appear in the appendix.

Before we consider the problem of estimating $\lambda, \mu, \alpha$ and $\beta$, it is worth collating the assumptions inherent in our model, and some of their implications.

\subsection{Model assumptions}

1. fire and rainfall events are independent of each other

2. fire and storm sizes/shapes are both iid

3. fires and storms are uniformly distributed in space

4. the rate at which fire and storm events occur is constant

For Assumption 1 we need to consider dependence in time and space. At the time scale of the model, temporal independence between fires and storms is reasonable. While the fire is actually burning it may affect local precipitation, but this is only a short term effect compared to the time for which burnt landscape is susceptible to erosion events. Perhaps more important is dependence caused by the geography, which will effect patterns of burning and precipitation. This dependence will effect the shape of fires and storms (grains) as the degree of burning or intensity of rainfall may be different upslope or downslope, for example. There will also be large scale (germ) effects on the location of fires (more frequent in mountainous areas, for example) and storms. By ignoring large scale effects, we are assuming that the catchment area is topographically homogenous (which is not to say flat, but the same type throughout). This means that our fitted model parameters will be specific for the type of catchment being modeled. Local geography could affect the intersections of fires and storms. Practically, ignoring such affects means that our risk measure, $\mathrm{E}\|R\|$, could also have a local component, in that the rate at which risk is converted to actual erosion events depends on the type of landscape the catchment is situated in.

Assumption 2 is saying that the local geography is homogenous across the catchment, so that the shapes of fires or storms are statistically similar from one end to the other. Independence of the grains also means that fires/storms do not interact if they overlap in space and time. Clearly this is not the case, but it is not a 
problem in practice, as we are ultimately only interested in the union of all burnt/stormy areas. Assumption 3 is saying that there are no large scale differences in weather/vegetation patterns across the catchment.

Assumption 4 is about seasonality. That is, we are supposing that there are no seasonal patterns in fire and storm events. This is clearly not the case, but we argue that the model will still give useful results. The reason is that burnt areas remain susceptible to erosion events for a long period of time, in the order of a couple of years. Thus, even though there will be seasonal patterns to fires, storms and high-magnitude erosion events, we can in effect spread them out over the year. The practical implication is that we need to ensure that the rates we use for fires and storms are annual rates.

\subsection{Model parameters}

Rainfall. Germ-grain models can be used to represent storms as discs with random intensity, size, velocity and duration (Cox \& Isham, 1988). More accurate models use clusters of disks as the grains (Cowpertwait, 1995), and can be extended to accommodate orographic effects (spatial nonstationarity) and seasonality (temporal nonstationarity) (Burton, et al., 2010). However these models require detailed information on the spatial and temporal distribution of rainfall over long time scales, in order to be parameterized and tested. In our case this data is currently unavailable and we therefore derived rainfall parameters using data from the Australian Bureau of Meteorology (BoM).

Let $r(t, x, y)$ be the rainfall intensity at time $t$ and spatial co-ordinates $(x, y)$, and define, for duration $h$ (in years) and area $A$,

$$
\begin{aligned}
R_{k}(h ; x, y) & =h^{-1} \int_{k h}^{(k+1) h} r(s, x, y) d s \\
R_{k}(h ; A) & =\|A\|^{-1} \iint_{A} R_{k}(h ; x, y) d x d y
\end{aligned}
$$

That is, $R_{k}(h ; x, y)$ is the average rainfall intensity at $(x, y)$ over the time period $(k h,(k+1) h)$, and $R_{k}(h ; A)$ is the rainfall intensity averaged over the time period $(k h,(k+1) h)$ and over the area $A$. Note that here $\|A\|$ is the area of $A$, rather than the volume. Let $R(h ; x, y)$ and $R(h ; A)$ denote randomly sampled values of $R_{k}(h ; x, y)$ and $R_{k}(h ; A)$. Let $f$ be a frequency (in years), and $A$ an area centered at $(x, y)$, then the BoM provides functions $\phi$ and $\xi$ such that

$$
\begin{aligned}
\mathrm{P}(R(h ; x, y)>\phi(h ; f, x, y)) & =h / f \\
\mathrm{P}(R(h ; A)>\phi(h ; f, x, y) \xi(\|A\| ; x, y)) & =h / f
\end{aligned}
$$

$\phi$ are called rainfall intensity-frequency-duration (IFD) curves (Figure 2a) and $\xi$ is called a depth-areareduction factor (DARF) (Figure 2b).
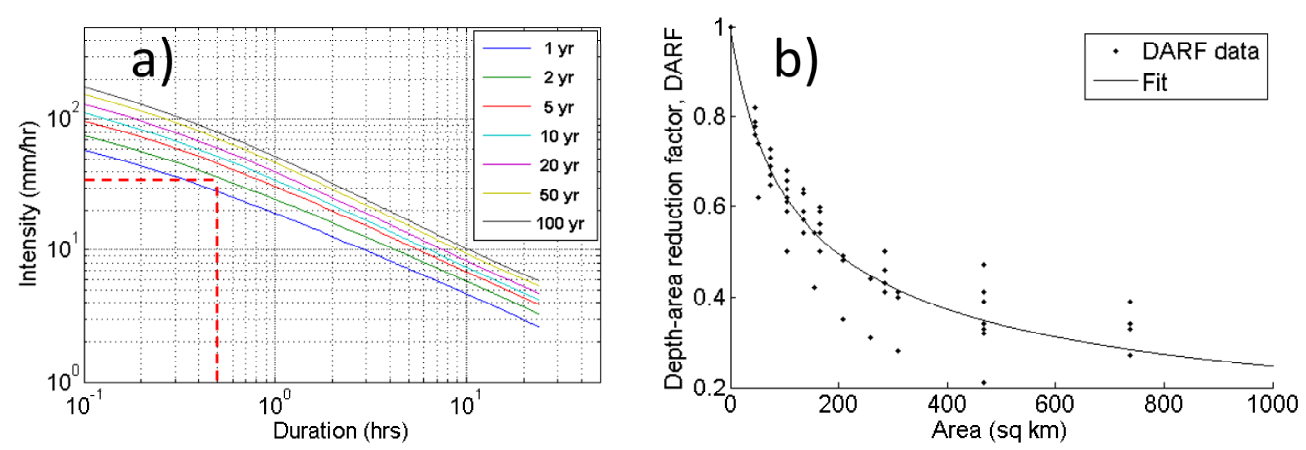

Figure 2. a) Intensity-frequency-duration curves for Bright, northeast Victoria (source: Australian Bureau of Meteorology). The 30-minute intensity threshold is shown by the red dashed line. b) Depth area reduction factor (DARF) for convective thunderstorms derived from radar data (Olivera, et al., 2008) (Texas) and (Curtis, 2011) (Colorado).

Debris flows in the eastern Victorian uplands are triggered by rainfall events with a 30-minute rainfall intensity of at least $35 \mathrm{~mm} \mathrm{~h}^{-1}$ (Nyman, et al., 2011). Thus for the model we restrict our attention to 30-minute 
storms that exceed this intensity threshold. Storm duration $h$ is therefore constant, and when expressed in years we get $h=(365 \times 24 \times 2)^{-1}$ years. Intense storm cells tend to be small, so we take an area of size $\|A\|=10 \mathrm{~km}^{2}$. Thus the frequency of these events, $f$, satisfies

$$
\phi\left(h=(365 \times 24 \times 2)^{-1} ; f\right) \xi(\|A\|=10)=35 m m h^{-1} .
$$

This gives $f=2.8$ and thus $\mu=(1 /\|A\|) \times(1 / f)=0.0357$ and $\beta=h\|A\|=1 / 1752=0.00057$.

Fire. If you ignore the shapes of grains in a germ-grain model and just look at their size, you get a compound Poisson process, and these can be found in the literature as fire models (Podur, et al., 2010). We use historical fire data from Victoria (VIC, 1072-2009) to test the assumptions underlying the compound Poisson model, and estimate the rate $\lambda$ and mean size $\alpha$ of fires. Note that the data is confined within state boundaries, which are arbitrary in the context of fire regimes. Preferably the fire regimes should be quantified for homogenous landscape units or eco-regions.

Within fire seasons the interarrival times for fires in Victoria were exponentially distributed (Figure 3a). The distribution of fire sizes displays power law behavior (Figure 3b). This suggests a Pareto distribution, though note that two or three parameter Weibull distributions have also been found to fit fire size data (Cui and Perera, 2008). We restricted the fires to those greater than $100 h a$ in size, as smaller fires are usually not intense enough to trigger high-magnitude erosion events. For these fires the rate of occurrence was $\lambda=1.18 \times 10^{-4}$ year $^{-1} \mathrm{~km}^{-2}$ and $4.18 \times 10^{-4}$ year $^{-1} \mathrm{~km}^{-2}$ for Victoria and the Australian Capital Territory respectively, highlighting the regional difference in fire regimes. Wildfires in the Australian Capital Territory are on average smaller and more frequent than in Victoria. The average size of Victorian bushfires over $100 \mathrm{ha}$ was $55 \mathrm{~km}^{2}$. Using a duration of two years (the time it takes the landscape to recover), we get $\alpha=2 \times 55=110$ years $\times \mathrm{km}^{2}$.
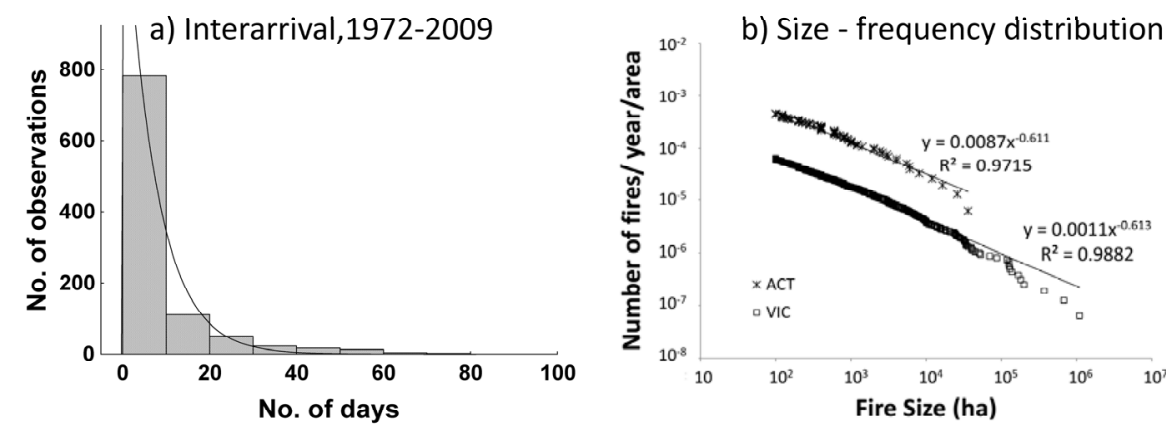

Figure 3. a) Inter-arrival time distributions for 1040 fires $>100 h a$ in Victoria. The arrival time was calculated from the start of one fire to the start of the next fire within fire seasons. b) The fire size-frequency distribution for Victoria (VIC) (1972-2009) and Australian Capital Territory (ACT) (1936-1999).

\section{DISCUSSION}

Fire and rainfall processes operate in the landscapes to produce a mosaic with erosion events occurring as "episodic patches of activity" (Miller, et al., 2003). Under this description, the patches are determined by intersection between storms and burn areas, and the activity (erosion) is determined by landscape attributes and the sensitivity to fire impacts. If the aim is to predict the likelihood of water quality impact following fire then the modeling effort should focus on activity (erosion) and how this changes with different rainfall inputs and fire severities. If the aim is to quantify risk within a catchment in context of, for example climate change, then the focus should be on the interaction between storms and burn areas.

In this paper we have shown how coverage processes provide a powerful framework within which the interaction of burnt areas and storms can be quantified. The expected area of intersection $\mathrm{E}\|R\|$ is a measure of hazard that is independent of the landscape vulnerability and the sediment transfer processes that occur following fire. It represents the average annual area $\left(\mathrm{km}^{2} \times\right.$ years $)$ where fire and rainfall satisfy the conditions known to be required for high-magnitude erosion events to occur in a particular landscape. Under this representation, the total risk is a function of both the hazard (patches or intersections) and the vulnerability (erosion and sediment transfer processes). Here, we were specifically interested in debris flows in Eucalypt forest of SE Australia and therefore used a known 30-minute rainfall threshold for post-fire debris flow initiation as a measure of vulnerability. Other thresholds may apply for different environments 
and processes. The strength of the model is that it provides a relative measure of hazard which responds directly to changes in fire disturbance and rainfall regimes.

A risk model expressed in this form has a number of obvious applications. To quantify the effect of climate change on the risk of high-magnitude erosion events, we need to quantify the effect of climate change on $\alpha$, $\beta, \lambda$ and $\mu$. A possible approach is to model the size and frequency of fires (and possibly also storms) as a function of the average annual temperature (or some other temperature based measure of fire hazard), which we can then project forward.

Another immediate application of the model is to quantify the effect of controlled burns. That is, we consider fires to be either small prescribed burns or large wildfires. As we increase the frequency of prescribed burns the frequency of wildfires will reduce. Provided we can quantify the relative frequencies of prescribed burns and wildfires, we can use the model to quantify the change in the risk of high-magnitude erosion events.

Finally we remark that the random sets we use in our germ-grain models for fires and storms can be very general. In particular, by thinking of them as higher dimensional objects we can include information such as the intensity of a storm, or the severity of a fire. We can even model recovery from fire disturbance by allowing the severity to decline over time.

\section{ACKNOWLEDGMENTS}

The project is funded by the Bushfire Cooperative Research Centre.

\section{REFERENCES}

Burton, A., H.J. Fowler, C.G. Kilsby and P.E. O'Connell (2010). A stochastic model for the spatial-temporal simulation of nonhomogeneous rainfall occurrence and amounts. Water Resources Research, 46(11), W11501.

Cannon, S.H., J.E. Gartner, M.G. Rupert, J.A. Michael, A.H. Rea and C. Parrett (2010). Predicting the probability and volume of postwildfire debris flows in the intermountain western United States. Geological Society of America Bulletin, 122(1-2), 127-144.

Cowpertwait, P.S.P. (1995). A Generalized Spatial-Temporal Model of Rainfall Based on a Clustered Point Process. Proc. R. Soc. Lond. A, 450(1938), 163-175.

Cox, D.R. and V. Isham (1988). A simple spatial-temporal model of rainfall. Proc. R. Soc. Lond. A, 415, 317-328.

Cui, W. and A.H. Perera (2008). What do we know about forest fire size distribution, and why is this knowledge useful for forest management? International Journal of Wildland Fire, 17(2), 234-244.

Flannigan, M.D., M.A. Krawchuk, W.J. de Groot, B.M. Wotton and L.M. Gowman (2009). Implications of changing climate for global wildland fire. International Journal of Wildland Fire, 18(5), 483-507.

Hall, P. (1988). Introduction to the Theory of Coverage Processes. Wiley.

Istanbulluoglu, E., D.G. Tarboton, R.T. Pack and C.H. Luce (2004). Modeling of the interactions between forest vegetation, disturbances, and sediment yields. Journal of Geophysical Research, 109(F01009).

Miller, D., C. Luce and L. Benda (2003). Time, space, and episodicity of physical disturbance in streams. Forest Ecology and Management, 178(1-2), 121-140.

Northrop, P. (1998). A clustered spatial-temporal model of rainfall. Proc. R. Soc. Lond. A, 454, 1875-1888.

Nyman, P., G.J. Sheridan, H.G. Smith and P.N. J. Lane (2011). Evidence of debris flow occurrence after wildfire in upland catchments of south-east Australia. Geomorphology, 125(3), 383-401.

Olivera, F., J. Choi, D. Kim and M.H. Li (2008). Estimation of average rainfall areal reduction factors in Texas using NEXRAD data. Journal of Hydrologic Engineering, 13(6), 438-448.

Podur, J.J., D.L. Martell and D. Stanford (2010). A compound Poisson model for the annual area burned by forest fires in the province of Ontario. Environmetrics, 21(5), 457-469.

Prosser, I.P. and L. Williams (1998). The effect of wildfire on runoff and erosion in native Eucalyptus forest. Hydrological Processes, 12(2), 251-265.

Robichaud, P.R., W.J. Elliot, F.B. Pierson, D.E. Hall and C.A. Moffet (2007). Predicting postfire erosion and mitigation effectiveness with a web-based probabilistic erosion model. CATENA, 71(2), 229-241.

Smith, H.G., G.J. Sheridan, P.N.J. Lane, P. Nyman and S. Haydon (2011). Wildfire effects on water quality in forest catchments: a review with implications for water supply. Journal of Hydrology, 396(1-2), 170192.

Whetton, P.H., A.M. Fowler, M.R. Haylock and A.B. Pittock (1993). Implications of climate change due to the enhanced greenhouse effect on floods and droughts in Australia. Climatic Change, 25(3), 289-317. 
White, I., A. Wade, M. Worthy, N. Mueller, T. Daniell and R. Wasson (2006). The vulnerability of water supply catchments to bushfires: Impacts of the January 2003 wildfires on the Australian Capital Territory. Australian Journal of Water Resources, 10(2), 1-16.

\section{APPENDIX}

Suppose that we have two independent Boolean models in $\mathrm{R}^{k}$. That is, let $\left\{\xi_{i}\right\}$ and $\left\{\zeta_{i}\right\}$ be independent stationary Poisson processes with intensities $\lambda_{\xi}$ and $\lambda_{\zeta}$, and let $\left\{X_{i}\right\}$ and $\left\{Y_{i}\right\}$ be mutually independent i.i.d. sequences of random sets, then our two models are $\mathcal{X}=\left\{\xi_{i}+X_{i}\right\}$ and $\mathcal{Y}=\left\{\zeta_{i}+Y_{i}\right\}$. Let $\Omega$ be a Borel subset of $\mathrm{R}^{k}$ then the intersection of $\Omega, \mathcal{X}$ and $\mathcal{Y}$ is given by $A=\Omega \cap\left(\cup_{i} \xi_{i}+X_{i}\right) \cap\left(\cup_{i} \zeta_{i}+Y_{i}\right)$. Let $\|A\|$ denote the content (Lebesgue measure) of $A$, then we have:

\section{Proposition}

If $\|\Omega\|<\infty$ then $\mathrm{E}\|A\|=\|\Omega\|\left(1-e^{-\lambda_{\xi} \mathrm{E}\|X\|}\right)\left(1-e^{-\lambda_{\zeta} \mathrm{E}\|Y\|}\right)$, where $X$ and $Y$ are random sets, distributed as the $X_{i}$ and $Y_{i}$ respectively. Moreover, let $\alpha_{X}(x)=\mathrm{E}\|(x+X) \cap X\|$ and $\alpha_{Y}(x)=\mathrm{E}\|(x+Y) \cap Y\|$, then

$$
\begin{aligned}
\operatorname{Var}\|A\|=\int_{\Omega} \int_{\Omega} & \left(\left(1-e^{-\lambda_{\xi} \alpha_{X}(0)}\right)^{2} e^{-2 \lambda_{\zeta} \alpha_{Y}(0)}\left(e^{\lambda_{\zeta} \alpha_{Y}(x-y)}-1\right)\right. \\
+ & \left(1-e^{-\lambda_{\zeta} \alpha_{Y}(0)}\right)^{2} e^{-2 \lambda_{\xi} \alpha_{X}(0)}\left(e^{\lambda_{\xi} \alpha_{X}(x-y)}-1\right) \\
& \left.+e^{-2\left(\lambda_{\xi} \alpha_{X}(0)+\lambda_{\zeta} \alpha_{Y}(0)\right)}\left(e^{\lambda_{\xi} \alpha_{X}(x-y)}-1\right)\left(e^{\lambda_{\zeta} \alpha_{Y}(x-y)}-1\right)\right) d x d y .
\end{aligned}
$$

\section{Proof}

Let $1_{\mathcal{X}}(x)=\left\{\begin{array}{ll}1 & x \in \cup_{i} \xi_{i}+X_{i} \\ 0 & \text { otherwise }\end{array}, 1_{\mathcal{Y}}(x)=\left\{\begin{array}{ll}1 & x \in \cup_{i} \zeta_{i}+Y_{i} \\ 0 & \text { otherwise }\end{array}\right.\right.$, then from Hall (1988) Equation (3.4) we have

$$
\begin{aligned}
\mathrm{E}\|A\| & =\mathrm{E} \int_{\Omega} 1_{\mathcal{X}}(x) 1_{\mathcal{Y}}(x) d x=\int_{\Omega} \mathrm{E} 1_{\mathcal{X}}(x) \mathrm{E} 1 \mathcal{Y}(x) d x \\
& =\int_{\Omega} \mathrm{P}(x \text { covered by } \mathcal{X}) \mathrm{P}(x \text { covered by } \mathcal{Y})=\int_{\Omega}\left(1-e^{-\lambda_{\xi} \mathrm{E}\|X\|}\right)\left(1-e^{-\lambda_{\zeta} \mathrm{E}\|Y\|}\right) \\
& =\|\Omega\|\left(1-e^{-\lambda_{\xi} \mathrm{E}\|X\|}\right)\left(1-e^{-\lambda_{\zeta} \mathrm{E}\|Y\|}\right) .
\end{aligned}
$$

Note that the result still holds when $\mathrm{E}\|X\|=\infty$ or $\mathrm{E}\|Y\|=\infty$.

For the variance we note first that

$$
\mathrm{E}\|A\|^{2}=\mathrm{E} \int_{\Omega} 1_{\mathcal{X}}(x) 1_{\mathcal{Y}}(x) d x \int_{\Omega} 1_{\mathcal{X}}(y) 1_{\mathcal{Y}}(y) d y=\int_{\Omega} \int_{\Omega} \mathrm{E} 1_{\mathcal{X}}(x) 1_{\mathcal{X}}(y) \mathrm{E} 1_{\mathcal{Y}}(x) 1_{\mathcal{Y}}(y) d x d y
$$

From Hall (1988) Equation (3.6) and preceding calculations

$\mathrm{E} 1_{\mathcal{X}}(x) 1_{\mathcal{X}}(y)=\mathrm{P}(x$ and $y$ covered by $\mathcal{X})$

$=1-\mathrm{P}(x$ not covered by $\mathcal{X})-\mathrm{P}(y$ not covered by $\mathcal{X})+\mathrm{P}($ neither $x$ nor $y$ covered by $\mathcal{X})$

$=1-2 e^{-\lambda_{\xi} \alpha_{X}(0)}+e^{-2 \lambda_{\xi} \alpha_{X}(0)+\lambda_{\xi} \alpha_{X}(x-y)}$

Thus

$$
\begin{aligned}
& \operatorname{Var}\|A\|=\mathrm{E}\|A\|^{2}-(\mathrm{E}\|A\|)^{2} \\
& =\int_{\Omega} \int_{\Omega}\left(\left(1-2 e^{-\lambda_{\xi} \alpha_{X}(0)}+e^{-2 \lambda_{\xi} \alpha_{X}(0)+\lambda_{\xi} \alpha_{X}(x-y)}\right)\left(1-2 e^{-\lambda_{\zeta} \alpha_{Y}(0)}+e^{-2 \lambda_{\zeta} \alpha_{Y}(0)+\lambda_{\zeta} \alpha_{Y}(x-y)}\right)\right. \\
& \left.\quad-\left(1-e^{-\lambda_{\xi} \alpha_{X}(0)}\right)^{2}\left(1-e^{-\lambda_{\zeta} \alpha_{Y}(0)}\right)^{2}\right) d x d y \\
& =\int_{\Omega} \int_{\Omega}\left(\left(1-e^{-\lambda_{\xi} \alpha_{X}(0)}\right)^{2} e^{-2 \lambda_{\zeta} \alpha_{Y}(0)}\left(e^{\lambda_{\zeta} \alpha_{Y}(x-y)}-1\right)+\left(1-e^{-\lambda_{\zeta} \alpha_{Y}(0)}\right)^{2} e^{-2 \lambda_{\xi} \alpha_{X}(0)}\left(e^{\lambda_{\xi} \alpha_{X}(x-y)}-1\right)\right. \\
& \left.\quad+e^{-2\left(\lambda_{\xi} \alpha_{X}(0)+\lambda_{\zeta} \alpha_{Y}(0)\right)}\left(e^{\lambda_{\xi} \alpha_{X}(x-y)}-1\right)\left(e^{\lambda_{\zeta} \alpha_{Y}(x-y)}-1\right)\right) d x d y .
\end{aligned}
$$

